\title{
Patterns of the Past. Tattoo Revival in the Cook Islands de Therese MANGOS et John UTANGA
}

\section{Sébastien Galliot}

\section{(2) OpenEdition}

\section{Journals}

\section{Édition électronique}

URL : http://journals.openedition.org/jso/6680

DOI : 10.4000/jso.6680

ISSN : $1760-7256$

\section{Éditeur}

Société des océanistes

\section{Édition imprimée}

Date de publication : 30 juin 2012

Pagination : 163-164

ISBN : 978-2-85430-032-1

ISSN : 0300-953x

\section{Référence électronique}

Sébastien Galliot, «Patterns of the Past. Tattoo Revival in the Cook Islands de Therese Mangos et John UTANGA », Journal de la Société des Océanistes [En ligne], 134 | 1er semestre 2012, mis en ligne le 29 juin 2012, consulté le 22 septembre 2020. URL : http://journals.openedition.org/jso/6680 ; DOI : https:// doi.org/10.4000/jso.6680

Ce document a été généré automatiquement le 22 septembre 2020.

(c) Tous droits réservés 


\title{
Patterns of the Past. Tattoo Revival in the Cook Islands de Therese MANGOS et John UTANGA
}

\author{
Sébastien Galliot
}

\section{RÉFÉRENCE}

MANGoS Therese et UTANGa John, 2011., Auckland, Punaroa Productions, 221 pages, bibliogr., glossaire, index, carte, environ 250 illustrations.

1 Partie d'une fascination pour un groupe de tatoueurs qu'elle observa lors du Festival des Arts du Pacifique aux îles Cook en 1992, l'auteure Therese Mangos dit avoir fait ce livre pour remédier à un manque d'information sur la question du tatouage dans cet archipel polynésien. Ainsi, cet ouvrage destiné au grand public est comparable ce qui a été écrit ces dernières années sur le tatouage d'autres sociétés polynésiennes (Allen 2006, Te Awekotuku \& Nikora 2008) en ce qu'il est construit sensiblement de la même manière. L'ouvrage composé de douze chapitres peut être découpé en trois grandes sections. Dans une première section (pp. 20-71) se trouvent un compte-rendu sur le mythe d'origine, une brève présentation du tatouage, de l'organisation sociale et de la cosmologie préchrétienne, une restitution des remarques faites durant les premiers contacts, ainsi qu'une brève analyse du rôle des missionnaires dans la disparition du tatouage. Une seconde section (pp. 72-101) consacrée à ce que les auteurs appellent la «renaissance » s'attache à présenter les principaux acteurs de ce fragile renouveau culturel. La troisième section, semblable à un catalogue d'exposition, consiste en une étude détaillée de motifs ornementaux anciens et modernes à partir de divers éléments de culture matérielle (nattes, étoffes d'écorce, sculpture, tatouage).

2 Le premier chapitre retrace la quête mythique d'Ina à la recherche du dieu Tinirau. Dans ce mythe de l'île méridionale de Mangaia, le tatouage fait son apparition sous la forme des traces de coups qu'Ina laisse sur ses montures successives (différents types 
de poissons de récifs) avant de rencontrer Makea le requin qui l'emmènera jusqu'à sa destination finale.

3 Les chapitres 2 et 3, respectivement intitulés The Cook Islands et Early Society, décrivent une société hiérarchique organisée autour des catégories ariki, mata'iapo, rangatira et ta'unga, au sein desquelles le principe de séniorité et les accomplissements individuels conditionnaient l'accumulation de mana. En ce qui concerne la cosmologie, les auteurs puisent largement dans l'ethnographie que le missionnaire William Wyatt Gill fit de l'île de Mangaia à la fin du xix ${ }^{\mathrm{e}}$ siècle. Le caractère contagieux du mana et la matérialisation d'esprits dans des espèces animales diverses sont mentionnés au passage afin de suggérer un lien entre les cultes préchrétiens voués aux divinités comme Tonga'iti et leur éventuelle représentation sous forme de motifs tatoués. Sur les experts tatoueurs ta'unga tātatau, un seul paragraphe de dix lignes nous rappelle qu'ils appartenaient à la classe bien connue des artisans et des prêtres. Le chapitre 4 intitulé tâtatau décrit l'outillage ancien (fragment d'os et pigment végétal) et souligne la rareté et l'imprécision des données. Pour cette raison, dans la suite du texte, les auteurs paraphrasent largement Te Rangi Hiroa qui n'avait rencontré que quelques rares personnes tatouées lors de sa visite en 1909. Semblant donner une place centrale à la pratique du tatouage et à ses motifs ornementaux, le titre de ce bel ouvrage est trompeur et le lecteur se rend compte assez vite du petit nombre des données disponibles sur le sujet. Le cinquième chapitre, Early Encouters, retrace les différents voyages à la suite desquels mention fut faite de la présence du tatouage, principalement dans les îles du Sud. L'intérêt ici est que la documentation du tatouage aux îles Cook semble avoir souffert de ce que les explorateurs étaient déjà habitués à cette pratique qu'ils avaient observée dans les groupes précédemment abordés. Ce qui expliquerait pourquoi le tatouage ne semble pas avoir suscité beaucoup d'intérêt lors des premiers contacts. Le processus d'évangélisation relativement rapide, la faible résistance des insulaires au message chrétien allèrent de pair, comme ailleurs, avec la mise en place de lois, (aux Cook ce furent les Blues Laws) interdisant le tatouage. C'est d'ailleurs accompagné d'un groupe d'Aitutaki fraîchement converti que John Williams inaugura son entreprise missionnaire aux îles Samoa.

À partir d'extraits des récits d'exploration (Cook, Blight et Kotzebue), le sixième chapitre établit une distinction entre la pratique de la scarification au nord de l'archipel et celle du tatouage au Sud.

5 La deuxième section de l'ouvrage se penche sur le tatouage contemporain et la renaissance de cette pratique. Les auteurs font remonter l'origine de cette renaissance en 1992, année du sixième Festival des Arts du Pacifique Sud qui eut lieu à Rarotonga. Construit autour du thème de la navigation traditionnelle, cet événement rassembla également un certain nombre de tatoueurs polynésiens qui ont vraisemblablement suscité des vocations auprès de la population locale. Dommage toutefois que la genèse de ce renouveau ne soit pas décrite plus en détail. On aurait notamment aimé connaître l'identité des tatoueurs étrangers (la présence de Raymond Graffe est évoquée) et les mécanismes qui expliquent ce regain d'intérêt pour le tatouage aux îles Cook. Le texte se poursuit avec cinq portraits (quatre tatoueurs et une jeune tatoueuse) des acteurs qui portent ou ont porté ce renouveau. Si leur parcours individuel est abordé, en revanche leurs réalisations dans le domaine du tatouage, et pour certains de la sculpture, ne sont illustrées que par quelques photos. 
6 Les cinq derniers chapitres sont consacrés aux motifs ornementaux des îles Aitutaki, Mangaia, Ngā Pū Toru et Rarotonga. À ce stade de l'ouvrage, les auteurs semblent n'avoir eu d'autre choix que d'élargir leur propos tant les données sur le tatouage sont rares. Sur ce thème précis, ils sont d'ailleurs contraints de paraphraser d'anciennes publications de Te Rangi Hiroa. Néanmoins, leur analyse comparative des motifs de tatouage, des objets de culte, des décorations des vaka, des nattes, des étoffes d'écorces s'avère intéressante et se traduit par la recherche de correspondance ou, comme aurait dit Gell (2010 [1998]) d'« axes de cohérence », dans ces différents domaines. Si ce travail reste très descriptif et ne propose pas de définition formelle du style ornemental des îles Cook, il examine avec précision l'éventail des motifs, leur application à différents supports de même que leur valeur de référence pour la création de nouveaux motifs.

7 C'est en fin de compte le tatouage qui est le parent pauvre de cette section car aux îles Cook comme ailleurs en Polynésie (si l'on excepte Samoa, les Marquises et la NouvelleZélande), le sujet aujourd'hui très populaire, n'a pas occupé une grande place dans les récits d'exploration. Et les ethnographes « urgentistes » envoyés sur le terrain par les musées de Hamburg et d'Hawaii au début du $\mathrm{xx}^{\mathrm{e}}$ siècle se bornèrent la plupart du temps à collecter des données de seconde main et des témoignages, et à relever quelques motifs tatoués sur la peau d'une poignée de personnes âgées. Malheureux également le terme de revival, lorsque l'on s'aperçoit que le prétendu renouveau culturel ne repose aujourd'hui que sur trois talentueux praticiens, Croc Coulter, Clive Nicholas et Teteni Pepeko.

8 S'il n'est pas le fruit d'universitaires mais d'auteurs passionnés par leur pays d'origine, Patterns of the Past présente néanmoins les résultats d'un travail de recherche sérieux, soigneusement mis en pages et richement illustré. Il vient en outre enrichir les monographies sur le tatouage océanien qui paraissent régulièrement depuis les années 1990 (Ottino-Garanger 1998, Kuwahara 2005, Allen 2006, Spenneman 2009, Te Awekotuku \& Nikora 2010). Comme Therese Mangos l'indique elle-même, il n'est pas exhaustif et présente en toute modestie le début de son exploration sur le tatouage ainsi que certaines de ses hypothèses. Il n'impressionnera probablement pas les anthropologues océanistes mais trouvera une place légitime dans la bibliothèque des passionnés de tatouage, ainsi que parmi le grand public.

\section{BIBLIOGRAPHIE}

ALLEN Tricia, 2006. Tattoo Traditions of Hawaii, Honolulu, Mutual Publishers.

GELL Alfred, 2010. L'art et ses agents, une théorie anthropologique, Grenoble, Les Presses du réel (traduction de Art \& Agency. An anthropological theory, Oxford, Clarendon Press, 1998).

KuwAHARA Makiko, 2005. Tattoo, An Anthropology, New-York, Berg Publishers.

OTTINO-GARANGER Pierre \& Marie-Noëlle, 1998. Le tatouage aux îles Marquises, s. l., Ch. Gleizal éditeur. SPENNEMAN Dirk H. R., 2009. Tattooing in the Marshall Islands, Honolulu, Bess Press. 
TE AWEKOTUKU N. \& L. WAIMARIE NIKORA, 2010. Mau Moko. Le monde du tatouage māori,Tahiti, Au Vent des îles. 